\title{
Extracranial-Intracranial High-Flow Bypass with Internal Carotid Artery Ligation at Neck for Giant Cavernous Internal Carotid Artery Dissecting Aneurysm in a Teen Girl with Postoperative Unusual Complication of Mono-ocular Blindness: A Case Report
}

\author{
Forhad H. Chowdhury' Sheikh Mohammod Ekram
Mohammod Shamsul Arifin ${ }^{2}$
1Department of Neurosurgery, National Institute of Neurosciences
and Hospital, Shere-e-bangla Nagar, Dhaka, Bangladesh \\ ${ }^{2}$ Department of Neurosurgery, Bangabandhu Sheikh Muzib Medical \\ University, Dhaka, Bangladesh
}

Address for correspondence Forhad Hossain Chowdhury, FCPS, Assistant Professor, Department of Neurosurgery, National Institute of Neurosciences and Hospital, Shere-e-bangla Nagar, Dhaka, 1207 , Bangladesh (e-mail: forhadchowdhury74@yahoo.com).

\begin{abstract}
Keywords

- extracranial-intracranial high-flow bypass

- giant cavernous internal carotid artery dissecting aneurysm

- radial artery graft

- mono-ocular blindness

Extracranial-intracranial (EC-IC) high-flow bypass with internal carotid artery (ICA) ligation at the neck is an important treatment option for giant cavernous ICA dissecting aneurysm. Such treatment may be associated with many complications. We report a case that developed postoperative mono-ocular blindness. So far to our knowledge such complication has not been reported in the literature. A 14-year-old girl presented with recent headache, eye ache, occasional double vision, and occasional right-sided visual impairment. Neurologic examination revealed no abnormality. Computed tomographic (CT) scan of the head showed a large, right parasellar, and mostly hyperdense space-occupying lesion. CT angiogram of the brain showed giant dissecting aneurysm of right-sided cavernous segment ICA. A right-sided external carotid artery-radial artery graft-middle cerebral artery (ECA-RAG-MCA [M2]) high-flow bypass was done with ligation of the right ICA in the neck. Postoperatively, the patient developed complete, mono-ocular, and permanent blindness on right side. Mono-ocular blindness is a serious complication from ophthalmic/central retinal artery thrombosis in high-flow EC-IC bypass with ICA ligation. Strategy is required to find and to set for its prevention.
\end{abstract}

\section{Introduction}

Developed by Professor Gazi Yasargil in 1967, cerebral revascularization has become an indispensable tool for managing patients with steno-occlusive intracranial ischemic disease, complex intracranial aneurysms that require deliberate occlusion of a parent artery and invasive skull base tumors. ${ }^{1-4}$ Patients with complex, giant aneurysm have a 5-year mortality rate (80\%). This means that they have a worse prognosis. ${ }^{1,5}$
As they cannot be treated by endovascular means, surgical clipping is the only option. Clipping is usually not performed because of a wide neck, a fusiform aneurysm, major parent vessels arising from the dome or the neck of the aneurysm, and because the aneurysm must be decompressed under direct trapping, all of which necessitate some form of bypass. ${ }^{1,6}$ It is well recognized that extracranial-intracranial (EC-IC) high-flow bypass (HFB) with therapeutic internal carotid artery (ICA) occlusion has become a treatment option received

December 15, 2018

accepted

February 18, 2019

published online

September 20, 2019
DOI https://doi.org/

10.1055/s-0039-1697729

ISSN 2277-954X.
C2019 Neurological Surgeons'

Society of India
License terms

() (1) $\Theta \circledast$ 
for giant ICA aneurysms. ${ }^{7}$ Such treatment may be associated with many complications such as graft occlusion, hemorrhage, infarct, ophthalmoplegia, hyperperfusion syndrome, cognitive disturbance, etc. We report a case that developed postoperative mono-ocular blindness after having EC-IC HFB with ICA ligation at the neck. So far to our knowledge, such complication has not been reported in the literature.

\section{Case Report}

A 14-year-old right-handed girl presented with recent headache, eye ache, occasional double vision, and occasional right-sided visual impairment for the past 2 months. Neurologic examination revealed no abnormality, including visual acuity, visual field, ocular movements, and funduscopy. Allen's test in both sides was negative. All other systemic examinations were within normal limit. Computed tomographic (CT) scan of the head showed a right parasellar, large, mostly hyperdense space-occupying lesion (-Fig. 1). $\mathrm{CT}$ angiogram of the brain showed giant dissecting aneurysm of right whole cavernous segment of ICA ( - Fig. 2). After counseling with the patient party, a right-sided external carotid artery-radial artery graft-middle cerebral artery (ECA-RAG-MCA [M2]) HFB was planned with ligation of ICA at its origin in the neck.

\section{Operation}

Under general anesthesia with endotracheal intubation, the patient was placed in supine position. Head was fixed with three-pin head holder with neck extension and head turning to the opposite side (30 degrees). Left upper limb was placed on a side "limb rest" in extended elbow for radial artery (RA) procurement.

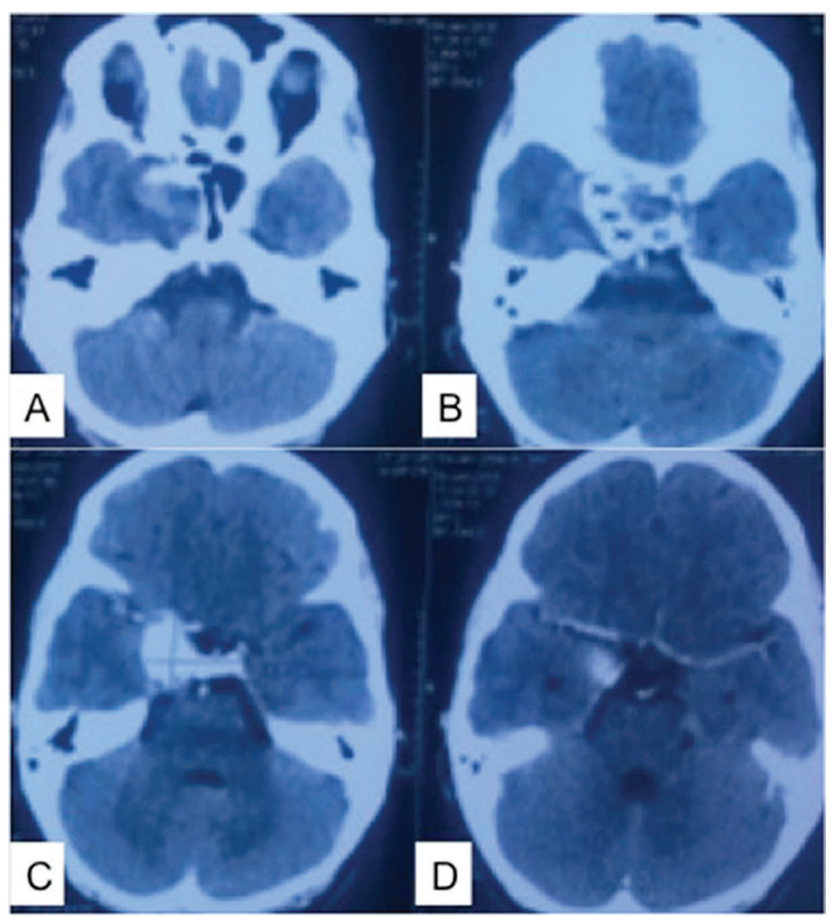

Fig. 1 (A-D) Initial CT scan of the head sequential axial sections showing probable right internal carotid artery giant aneurysm.

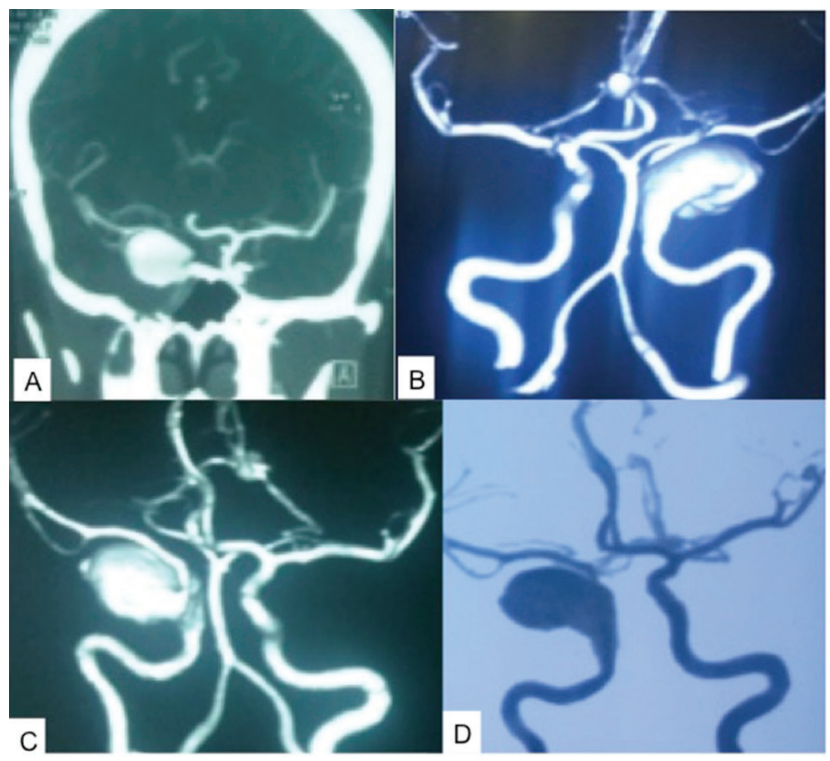

Fig. 2 (A-D) CTA of the brain showing right cavernous ICA giant fusiform aneurysm.

With longitudinal incision, the RA was harvested from brachial bifurcation at elbow to wrist $(20 \mathrm{~cm})$. The artery was distended, and then it was kept in heparin and papaverine-mixed normal saline. The forearm wound was closed with a drain.

A curve incision on the right side of the neck was made from the mastoid tip and extended downward and medially $2 \mathrm{~cm}$ posterior to the angle of mandible to the midline. Sternocleidomastoid muscle was retracted laterally. With further dissection, posterior belly of the digastric muscle, hypoglossal nerve, internal jugular vein, common carotid, ICA, and ECA with its branches were identified.

A right-sided precoronal post hairline curvilinear incision was made, and superficial temporal artery (STA) and its parietal branch were procured and prepared for STA-MCA insurance bypass as donor artery. A temporally extended pterional craniotomy was done. Temporal bone was removed down to the middle fossa floor. A $26 \mathrm{~F}$ thoracostomy tube was passed from cervical wound to the middle fossa floor. RAG was passed from middle fossa floor to cervical wound through the tube.

After durotomy, an STA-MCA (temporal M4) "insurance bypass" was done. After sylvian dissection, temporal M2 was identified and prepared for bypass. RAG and temporal M2 bypass was made after systemic heparinization.

With the control of ECA just distal to bifurcation of common carotid artery (CCA), an anastomosis was made between caudal end of RAG and ECA. The patency and flow through the anastomoses and RAG were checked with micro Doppler. ICA was permanently ligated with 1-0 silk at its origin in the neck. Cervical wound and craniotomy wound were closed with drains.

\section{Postoperative Course}

The patient recovered well from anesthesia, but she developed right-sided complete ophthalmoplegia with ptosis. 
After elevation of right-sided upper eyelid, her vision was checked. Initially she said that she could not see with her right eye, but later she confirmed that she could. However, there was blurring of vision on right side. Funduscopy revealed pale fundus. Postoperatively, the patient was on aspirin and inj. heparin. CT scan on first postoperative day (POD) showed no infarct or any gross hematoma ( - Fig. 3). CT angiogram on second POD showed right ECA-RAG-M2 bypass with absence of the right ICA with aneurysm ( - Fig. 4). ICA seemed to be occluded up to its terminal bifurcation with absent right-sided ophthalmic artery (-Fig. 5). On second POD, check examination showed no vision in right eye as well as absence of perception of light (PL) and projection of light (PR), but funduscopic examination revealed no definite abnormality except pallor. Her ophthalmoplegia with ptosis recovered completely within 4 weeks after operation, but there was no PL and PR in right side with optic atrophy till last follow-up (8 months after operation).

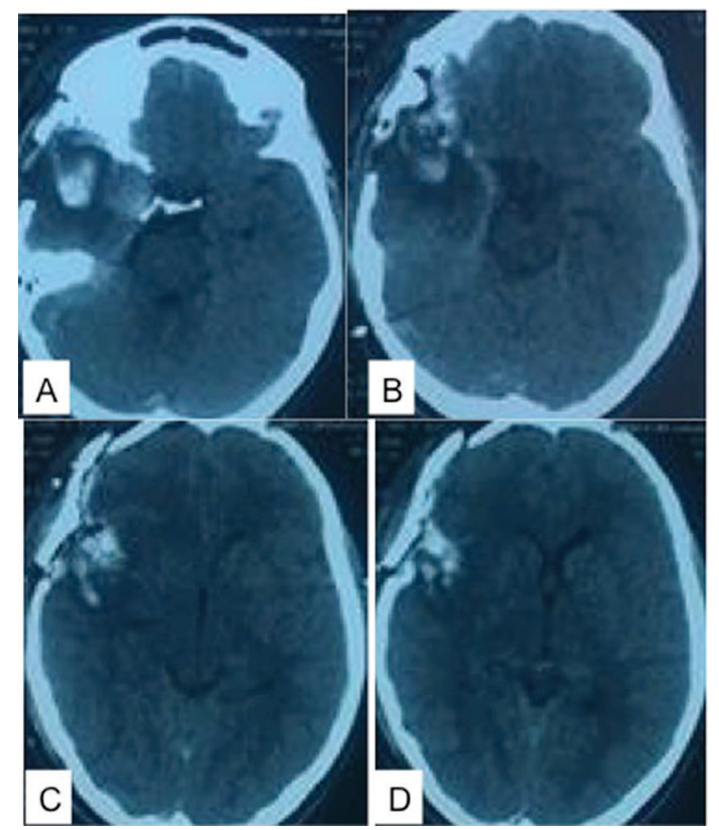

Fig. 3 Postoperative CT scan of the head axial images (A-D) on first POD showing no gross infarct or hematoma.

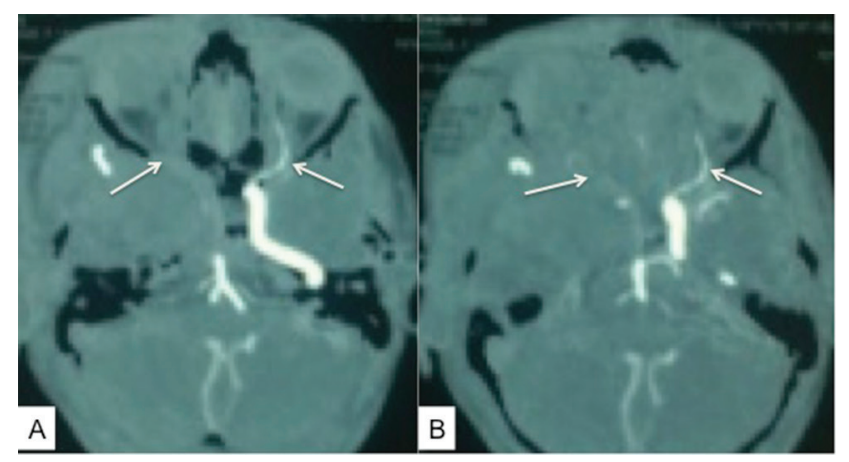

Fig. 4 CTA of the brain axial images (A, B) on second POD showing presence of left ICA and left ophthalmic artery (arrow mark) and absent of aneurysm, right ICA, and right ophthalmic artery (arrow mark).

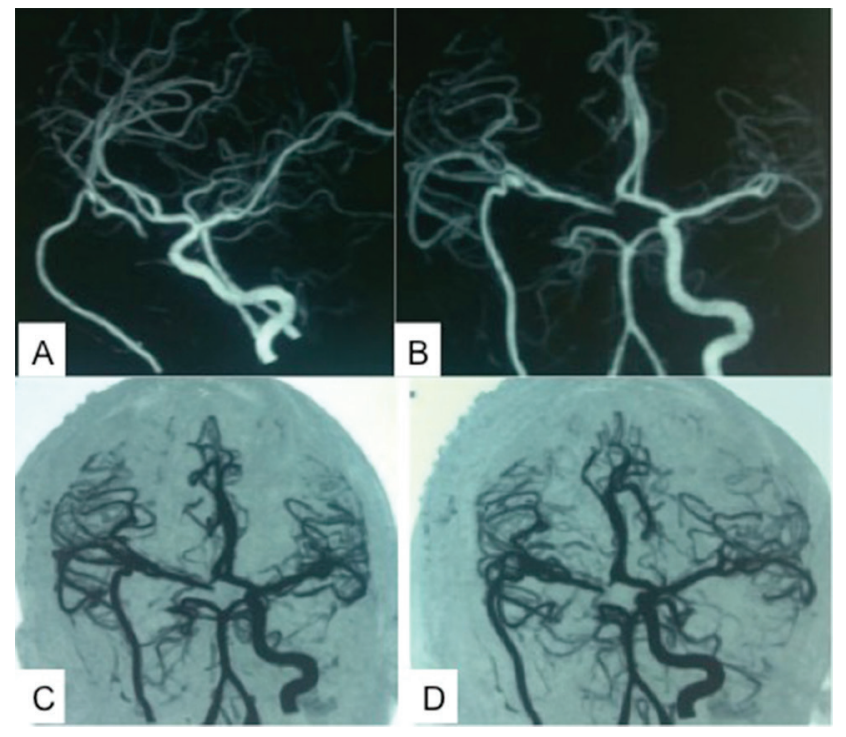

Fig. 5 CTA of the brain (A-D) on second POD showing presence of left ICA and absent of aneurysm, right ICA with patent right-sided high-flow EC-IC bypass.

\section{Discussion}

The credit for the first intracranial EC-IC bypass surgery goes to Donaghy and Yasargil in 1967. ${ }^{8}$ Lougheed then performed the first HFB 2 years later., ${ }^{9,10}$ There are two major indications for EC-IC bypass surgery: (1) flow augmentation and (2) flow replacement. ${ }^{7}$

High-flow bypass followed by ligation of the ICA is an effective treatment, but the impact of abrupt occlusion of the ICA is unpredictable, especially on postoperative cognitive function. HFB followed by ICA ligation can achieve good clinical outcomes. This HFB (flow from the ECA to MCA via the RAG) cures the cavernous and supraclinoidal ICA aneurysm and usually does not allow anterograde flow into the aneurysm. ${ }^{11}$

For HFB, RAG or great saphenous vein graft is usually used. ${ }^{1,7}$

The RA has the benefits of having a thick wall, a good size match to the $\mathrm{M} 2$, and providing an intermediate flow that has been proven by many authors to be ideal to the brain. ${ }^{12}$ The RA also has a proven long-term patency rate that has been accepted as superior to great saphenous vein grafts. Pressure distension technique as described by Ramanathan et al can effectively prevent spasm. ${ }^{12}$

There is a general agreement, to use end-to-side anastomosis as the main form of anastomosis for most cases of cerebral revascularization. ${ }^{1} \mathrm{~A}$ standard end-to-side anastomosis technique with two anchoring stitches at the heel and at the toe is usually used. ${ }^{1}$ Either slit or tear drop arteriotomy can be used during anastomosis. ${ }^{1,12}$

Studies on the short- and long-term patency of HF EC-IC bypass grafts have shown that for the experienced neurovascular surgeon, patencies of 90 to $95 \%$ can be obtained. ${ }^{7}$ In a study of 137 revascularizations, Sekhar and Kalavakonda reported an overall graft patency rate of $95.6 \% .^{13,14}$ 
STA-MCA bypass has its own share of complications, the major ones being graft occlusion, subgaleal hematoma, scalp necrosis, and postoperative intracranial hemorrhage. ${ }^{1}$ Graft occlusion can be prevented by meticulous dissection of both the donor and recipient arteries with minimal handling of the endothelium. Rarely post bypass hyperperfusion syndrome can develop, which proved to be very difficult to treat. ${ }^{9}$

In a study of 137 revascularizations, Sekhar and Kalavakonda ${ }^{13,14}$ reported an incidence of postoperative cerebral infarction of $16.8 \%$. In addition to graft thrombosis, cerebral infarction can be caused by a prolonged ischemia time of the recipient segment of the MCA. Chazono et $\mathrm{al}^{15}$ reported a case of intraoperative infarction with a clamping time of 65 minutes and no infarction in three identical cases with clamping times of 30 to 40 minutes.

The risk of ischemia in the donor artery territory is related to the clamp time, which is directly related to the learning curve. The risk can be reduced by raising the mean arterial pressure greater than $20 \%$ above baseline and reducing metabolism by burst suppression. ${ }^{1}$

Successful HFB using RAG with supportive STA-MCA bypass and ICA ligation does not adversely affect postoperative cognitive function. ${ }^{11}$

In our case, the patient became blind postoperatively on right side (no PL or PR), with normal left-sided vision. Her ophthalmoplegia recovered completely, but right-sided eye vision remained absent. There was no surgical manipulation in the areas related to intracranial ICA, ophthalmic artery, central retinal artery, and optic apparatus. ECA clamping time was less than 25 minutes. There was no drop of perioperative pressure. We think that this mono-ocular blindness occured due to ophthalmic artery/central retinal artery occlusion by progression of thrombosis from the ICA to the ophthalmic artery and then to central retinal artery, as it was supported by nonvisualization of the opthalmic artery on right side compared with the left one. Initially funduscopy revealed pallor fundus only (that can occur in cilioretinal sparing of central retinal artery occlusion). ${ }^{16}$ The thrombosis occurred when the patient was on inj. heparin and aspirin. Now the question is, how can we prevent it? Yes, we may think of one option, where we can stop thrombus propagation by trapping the ICA from the neck to clinoid ICA proximal to the ophthalmic artery (after clinoid drilling), but once again, it may be associated with ophthalmic artery spasm or very rarely in trauma. Now should we use trapping in all such cases or in which cases should we use trapping other than only neck ligation of ICA in the neck?

\section{Conclusion}

Mono-ocular blindness is a serious complication. It is very difficult to draw a conclusion from a single case; we have to learn more about the mono-ocular blindness from ophthalmic/central retinal artery thrombosis in EC-IC HFB with ICA ligation to prevent it.

\section{Conflicts of Interest}

None declared.

\section{References}

1 Biswas A, Samadoni AE, Elbassiouny A, Sobh K, Hegazy A. Extracranial to intracranial by-pass anastomosis: review of our preliminary experience from a low volume center in Egypt. Asian. J Neurosurg 2015;10(4):303-309

2 Amin-Hanjani S, Barker FG II, Charbel FT, Connolly ES Jr, Morcos JJ, Thompson BG; Cerebrovascular Section of the American Association of Neurological Surgeons. Congress of Neurological Surgeons. Extracranial-intracranial bypass for stroke-is this the end of the line or a bump in the road? Neurosurgery 2012;71(3):557-561

3 Dong Y, Teoh HL, Chan BP, et al. Changes in cerebral hemodynamic and cognitive parameters after external carotid-internal carotid bypass surgery in patients with severe steno-occlusive disease: a pilot study. J Neurol Sci 2012;322(1-2):112-116

4 Fiedler J, Přibáň V, Skoda O, Schenk I, Schenková V, Poláková S. Cognitive outcome after EC-IC bypass surgery in hemodynamic cerebral ischemia. Acta Neurochir (Wien) 2011;153(6):1303-1311, discussion 1311-1312

5 Kalani MY, Zabramski JM, Hu YC, Spetzler RF. Extracranial-intracranial bypass and vessel occlusion for the treatment of unclippable giant middle cerebral artery aneurysms. Neurosurgery 2013;72(3):428-435, discussion 435-436

6 Krylov VV, Nakhabin OIu, Polunina NA, et al. [High-flow extracranial-intracranial (EC-IC) bypass surgery in a patient with giant right cavernous ICA aneurysm] [in Russian]. Zh Vopr Neirokhir Im N N Burdenko 2012;76(5):40-46, discussion 47

7 Sia SF, Morgan MK. High flow extracranial-to-intracranial brain bypass surgery. J Clin Neurosci 2013;20(1):1-5

8 Yasargil MG, Stuttgart: Microsurgery Applied to Neurosurgery. Georg Thieme; 1969

9 Matano F, Murai Y, Tateyama K, et al. Perioperative complications of superficial temporal artery to middle cerebral artery bypass for the treatment of complex middle cerebral artery aneurysms. Clin Neurol Neurosurg 2013;115(6):718-724

10 Mohit AA, Sekhar LN, Natarajan SK, Britz GW, Ghodke B. Highflow bypass grafts in the management of complex intracranial aneurysms. Neurosurgery 2007;60(2, Suppl 1):ONS105ONS122, discussion ONS122-ONS123

11 Ono H, Inoue T, Tanishima T, Tamura A, Saito I, Saito N. Highflow bypass with radial artery graft followed by internal carotid artery ligation for large or giant aneurysms of cavernous or cervical portion: clinical results and cognitive performance. Neurosurg Rev 2018;41(2):655-665

12 Ramanathan D, Temkin N, Kim LJ, Ghodke B, Sekhar LN. Cerebral bypasses for complex aneurysms and tumors: longterm results and graft management strategies. Neurosurgery 2012;70(6):1442-1457, discussion 1457

13 Zhu W, Tian YL, Zhou LF, Song DL, Xu B, Mao Y. Treatment strategies for complex internal carotid artery (ICA) aneurysms: direct ICA sacrifice or combined with extracranial-to-intracranial bypass. World Neurosurg 2011;75(3-4):476-484

14 Sekhar LN, Kalavakonda C. Cerebral revascularization for aneurysms and tumors. Neurosurgery 2002;50(2):321-331

15 Chazono H, Okamoto Y, Matsuzaki Z, et al. Extracranial-intracranial bypass for reconstruction of internal carotid artery in the management of head and neck cancer. Ann Vasc Surg 2003;17(3):260-265

16 Hayreh SS, Zimmerman MB. Fundus changes in central retinal artery occlusion. Retina 2007;27(3):276-289 